\title{
Specimen records for North American Lepidoptera (Insecta) in the Oregon State Arthropod Collection. Pyraloidea: Crambidae Latreille 1810 and Pyralidae Linnaeus, 1758
}

Jon H. Shepard

Paul C. Hammond

Christopher J. Marshall

Oregon State Arthropod Collection, Department of Integrative Biology, Oregon State University, Corvallis OR 97331

Cite this work, including the attached dataset, as:

Shepard, J. S, P. C. Hammond, C. J. Marshall. 2019. Specimen records for North American Lepidoptera (Insecta) in the Oregon State Arthropod Collection. Pyraloidea: Crambidae Latreille 1810 and Pyralidae Linnaeus, 1758. Catalog: Oregon State Arthropod Collection 3(2) (beta version). http://dx.doi.org/10.5399/osu/cat osac.3.2.4596

\section{Introduction}

These records were generated using funds from the LepNet project (Seltmann et al. 2017) - a national effort to create digital records for North American Lepidoptera. The dataset published herein contains the label data for all North American specimens of Crambidae in the collection as of March 2019. This dataset is not complete, as portions of our North American Pyralidae remain to be digitized. This will be accomplished with a future dataset. A beta version of these data records will be made available on the OSAC server (http:/ / osac.oregonstate.edu/IPT) at the time of this publication. The beta version will be replaced in the near future with an official release (version 1.0), which will be archived as a supplemental file to this paper.

\section{Methods}

Basic digitization protocols and metadata standards can be found in (Shepard et al. 2018). Identifications for this family are tricky, especially in the Pacific Northwest where the pyraloid fauna remains relatively unstudied. As a result, providing determinations to species, or even genus, was not always possible. In the interest of making these specimens visible to taxonomists, we include even those specimens identified only to family. Generic and species determinations were reviewed prior to digitization by Jon Shepard and Paul Hammond using (Group 2019). Nomenclature follows that of the Digital Guide to Moth Identification (Group 2019).

\section{Results}

Crambidae: There are 3461 specimens identified at species-level (species or subspecies), with another 123 specimens identifiable only to genus. The following genera are present: Achroia Hübner, 1819, Achyra Guenée, 1849, Agathodes Guenée, 1854, Agriphila Hübner, 1825, Anageshna Munroe, 1956, Anania Hübner, 1923, Anatralata Munroe, 1961, Blepharomastix Lederer, 1863, Catoptria Hübner, 1825, Chalcoela Zeller, 1872, Choristostigma Warren, 1892, Chrysoteuchia Hübner, 1825, Clydonopteron Riley, 1880, Cosipara Munroe, 1972, Crambus Fabricius, 1798, Crocidophora Lederer, 1863, Desmia 
Westwood, 1832, Diaphania Hübner, 1818, Diastictis Hübner, 1818, Dicymolomia Zeller, 1872, Donacaula Meyrick, 1890, Euchromius Guenée, 1845, Eudonia Billberg, 1820, Eurrhypara Hübner, 1825, Evergestis Hübner, 1825, Fissicrambus Bleszynski, 1963, Frechinia Munroe, 1961, Fumibotys Munroe, 1976, Galleria Fabricius, 1798, Gesneria Hübner, 1925, Gyros Edwards, 1881, Hahncappsia Munroe, 1976, Herpetogramma Lederer, 1863, Loxostege Hübner, 1825, Macrotheca Ragonot, 1891, Mecyna Doubleday, 1849, Microcrambus Bleszynski, 1963, Microtheoris Meyrick, 1932, Mimoschinia Warren, 1892, Neodactria Landry, 1995, Noctueliopsis Munroe, 1961, Nomophila Hübner, 1825, Occidentalia Dyar \& Heinrich, 1927, Orenaia Duponchel, 1845, Ostrinia Hübner, 1825, Palpita Hübner, 1808, Parapeiacia Bleszynski, 1966, Parapoynx Hübner, 1825, Pediasia Hübner, 1825, Perispasta Zeller, 1875, Petrophila Guilding, 1830, Phlyctaenia Kützing, 1849, Pogonogenys Munroe, 1961, Procymbopteryx Munroe, 1961, Prorasea Grote, 1878, Pseudoschoenobius Fernald, 1891, Pyrausta Schrank, 1802, Saucrobotys Munroe, 1976, Scoparia Haworth 1811, Sitochroa Hübner, 1825, Spoladea Guenée, 1854, Tehama Hulst, 1888, Thaumatopsis Morrison, 1874, Tosale Walker, 1863, Udea Guenée, 1845, Uresiphita Hübner, 1825, Urola Walker, 1863 (Table 1).

Pyralidae: Of the pyralid specimens digitized at this time, only 24, belonging to the subfamily Phycitinae, could not be identified to genus; an additional 157 specimens could only be identified at the generic level. To date, 1383 specimens of North American Pyralidae were identified to specieslevel (species or subspecies) and digitized, belonging to the following genera: Acrobasis Zeller, 1839, Acrobasis Zeller, 1839, Acroncosa Barnes \& McDunnough, 1917, Aglossa Latreille, 1796, Alberada Heinrich, 1939, Ambesa Grote, 1880, Amyelois Amsel, 1956, Ancylosis Zeller, 1839, Bandera Ragonot, 1887, Dasypyga Ragonot, 1887, Dioryctria Zeller, 1846, Ephestia Guenée, 1845, Ephestiodes Ragonot, 1887, Etiella Zeller, 1839, Euzophera Zeller, 1867, Homoeosoma Curtis, 1833, Honora Grote, 1878, Hypsopygia Hübner, 1825, Interjectio Heinrich, 1956, Laetilia Ragonot, 1889, Lipographis Ragonot, 1887, Macalla Walker, 1859, Melitara Walker, 1863, Myelopsis Heinrich, 1956, Olybria Heinrich, 1956, Oneida Hulst, 1889, Phobus Heinrich, 1956, Phycitodes Hampson, 1917, Pima Hulst, 1888, Plodia Guenée, 1845, Pococera Zeller, 1848, Promylea Ragonot, 1887, Pyla Grote, 1882, Pyralis Linnaeus, 1758, Rostrolaetilia Blanchard \& Ferguson, 1975, Sarata Ragonot, 1887, Sciota Hulst, 1888, Toripalpus Grote, 1878, Vitula Ragonot, 1887, Zophodia Hübner, 1825 (Table 2).

Table 1. Crambidae

Taxon \#specimens

\begin{tabular}{|l|l|}
\hline Achroia grisella (Fabricius, 1794) & 17 \\
\hline Achyra occidentalis Packard, 1873 & 5 \\
\hline Achyra rantalis Guenée, 1854 & 7 \\
\hline Agathodes designalis Guenée, 1854 & 5 \\
\hline Agriphila anceps Grote, 1880 & 1 \\
\hline Agriphila attenuatus Grote, 1880 & 6 \\
\hline Agriphila biothanatalis Hulst, 1886 & 132 \\
\hline Agriphila plumbifimbriellus Dyar, 1904 & 79 \\
\hline Agriphila undatus Grote & 2 \\
\hline Anageshna primordialis Dyar, 1906 & 1 \\
\hline Anania funebris (Strom, 1768) & 7 \\
\hline Anania mysippusalis (Walker, 1859) & 8 \\
\hline
\end{tabular}


Table 1 (continued)

Taxon

\#specimens

\begin{tabular}{|c|c|}
\hline Anatralata versicolor Warren, 1892 & 1 \\
\hline Blepharomastix ranalis (Guenée, 1854) & 2 \\
\hline Catoptria latiradiellus Walker, 1863 & 2 \\
\hline Catoptria oregonicus Grote & 17 \\
\hline Chalcoela iphitalis Walker, 1859 & 15 \\
\hline Choristostigma elegantalis Warren, 1892 & 4 \\
\hline Chrysoteuchia topiarius Zeller, 1866 & 173 \\
\hline Clydonopteron undetermined & 1 \\
\hline Cosipara tricoloralis Dyar, 1904 & 27 \\
\hline Crambus agitatellus Clemens, 1861 & 2 \\
\hline Crambus dimidiatus Haworth, 1809 & 1 \\
\hline Crambus gausapalis Hulst, 1886 & 3 \\
\hline Crambus girardellus Clemens, 1860 & 1 \\
\hline Crambus leachellus Zincken, 1818 & 210 \\
\hline Crambus pascuella floridus Zeller, 1873 & 3 \\
\hline Crambus perlella innotatellus Walker, 1863 & 5 \\
\hline Crambus sperryellus Klots, 1940 & 20 \\
\hline Crambus tutillus McDunnough, 1921 & 129 \\
\hline Crambus unistriatellus Packard, 1868 & 44 \\
\hline Crambus undetermined & 24 \\
\hline Crocidophora serratissimalis Zeller, 1872 & 1 \\
\hline Desmia funeralis Hübner & 2 \\
\hline Desmia maculalis Westwood, 1831 & 13 \\
\hline Diaphania hyalinata (Linnaeus, 1767) & 1 \\
\hline Diaphania nitidalis (Stoll, 1781) & 2 \\
\hline Diastictis fracturalis (Zeller, 1872) & 7 \\
\hline Diastictis robustior Munroe, 1956 & 5 \\
\hline Diastictis sperryorum Munroe, 1956 & 1 \\
\hline Dicymolomia metalliferalis Packard, 1873 & 51 \\
\hline Donacaula melinellus Clemens, 1861 & 1 \\
\hline Donacaula undetermined & 1 \\
\hline Euchromius ocellea (Haworth, 1811) & 148 \\
\hline Eudonia commortalis Dyar, 1921 & 3 \\
\hline Eudonia echo Dyar, 1928 & 1 \\
\hline Eudonia leucophthalma Dyar, 1928 & 2 \\
\hline Eudonia rectilineata Li, Li \& Nuss, 2012 & 1 \\
\hline
\end{tabular}


Table 1 (continued).

Taxon

\#specimens

\begin{tabular}{|c|c|}
\hline Eudonia spenceri Munroe, 1972 & 18 \\
\hline Eudonia torniplagalis Dyar, 1904 & 1 \\
\hline Eurrhypara hortulata Linnaeus, 1758 & 5 \\
\hline Evergestis eurekalis Barnes \& McDunnough, 1914 & 7 \\
\hline Evergestis funalis (Grote, 1878 ) & 14 \\
\hline Evergestis pallidata (Hufnagel, 1767) & 22 \\
\hline Evergestis rimosalis Guenée, 1854 & 1 \\
\hline Evergestis simulatilis Grote, 1880 & 7 \\
\hline Evergestis subterminalis Barnes \& McDunnough, 1914 & 10 \\
\hline Fissicrambus mutabilis (Clemens, 1860) & 2 \\
\hline Frechinia helianthiales (Murt., 1897) & 1 \\
\hline Frechinia laetalis Barnes \& McDunnough, 1914 & 1 \\
\hline Fumibotys fumalis Guenée, 1854 & 13 \\
\hline Galleria mellonella (Linnaeus, 1758) & 72 \\
\hline Gesneria centuriella (Denis \& Schiffermuller, 1775) & 4 \\
\hline Gyros muiri Edwards, 1881 & 19 \\
\hline Hahncappsia coloradensis Grote \& Robinson, 1867 & 3 \\
\hline Herpetogramma abdominalis Zeller & 4 \\
\hline Herpetogramma pertextalis Lederer, 1863 & 174 \\
\hline Herpetogramma thestealis Walker, 1859 & 1 \\
\hline Loxostege anartalis Grote, 1877 & 9 \\
\hline Loxostege cereralis Zeller, 1872 & 30 \\
\hline Loxostege ephippialis (Zetterstedt, 1839) & 1 \\
\hline Loxostege indentalis (Grote, 1883) & 39 \\
\hline Loxostege lepidalis Hulst, 1886 & 79 \\
\hline Loxostege sierralis Munroe, 1976 & 12 \\
\hline Loxostege sticticalis (Linnaeus, 1761) & 15 \\
\hline Loxostege terpnalis Barnes \& McDunnough, 1918 & 2 \\
\hline Macrotheca ponda (Dyar, 1907) & 2 \\
\hline Mecyna mustelinalis Packard, 1873 & 66 \\
\hline Microcrambus elegans Clemens, 1860 & 2 \\
\hline Microtheoris ophionalis Walker, 1859 & 26 \\
\hline Mimoschinia rufofascialis (Stephens, 1834) & 21 \\
\hline Neodactria luteolellus Clemens, 1860 & 43 \\
\hline Noctueliopsis virula Barnes \& McDunnough, 1918 & 2 \\
\hline Nomophila nearctica Munroe, 1973 & 76 \\
\hline
\end{tabular}


Table 1 (continued)

Taxon

\#specimens

\begin{tabular}{|c|c|}
\hline Occidentalia comptulatalis Hulst, 1886 & 4 \\
\hline Orenaia arcticalis Munroe, 1974 & 1 \\
\hline Ostrinia nubilalis (Hubner, 1796) & 6 \\
\hline Ostrinia penitalis Grote, 1876 & 5 \\
\hline Palpita magniferalis Walker, 1861 & 9 \\
\hline Parapediasia teterrellus (Zincken, 1821) & 71 \\
\hline Parapoynx badiusalis Walker, 1859 & 9 \\
\hline Parapoynx obscuralis Grote, 1881 & 2 \\
\hline Pediasia browerellus Klots, 1942 & 1 \\
\hline Pediasia dorsipunctellus Kearfott, 1908 & 133 \\
\hline Pediasia trisecta Walker, 1856 & 145 \\
\hline Perispasta caeculalis Zeller, 1875 & 12 \\
\hline Petrophila avernalis Grote, 1878 & 3 \\
\hline Petrophila confusalis Walker, 1865 & 65 \\
\hline Phlyctaenia coronata Hufnagel, 1767 & 29 \\
\hline Pogonogenys proximalis Fernald & 2 \\
\hline Procymbopteryx belialis Druce, 1899 & 1 \\
\hline Prorasea praeia Dyar, 1917 & 2 \\
\hline Prorasea simalis Grote, 1878 & 9 \\
\hline Pseudoschoenobius opalescalis Hulst, 1886 & 10 \\
\hline Pyrausta acrionalis Walker, 1859 & 1 \\
\hline Pyrausta californicalis Packard, 1873 & 81 \\
\hline Pyrausta dapalis Grote, 1881 & 2 \\
\hline Pyrausta fodinalis Lederer, 1863 & 41 \\
\hline Pyrausta grotei Munroe, 1876 & 9 \\
\hline Pyrausta nexalis(Hulst 1886) & 6 \\
\hline Pyrausta laticlavia Grote \& Robinson, 1867 & 4 \\
\hline Pyrausta linealis Fernald, 1894 & 1 \\
\hline Pyrausta nicalis Grote, 1878 & 29 \\
\hline Pyrausta perrubralis Packard, 1873 & 38 \\
\hline Pyrausta pilatealis Barnes \& McDunnough, 1914 & 1 \\
\hline Pyrausta pseuderosnealis Munroe, 1976 & 1 \\
\hline Pyrausta semirubralis Packard, 1873 & 20 \\
\hline Pyrausta signatalis Walker, 1865 & 2 \\
\hline Pyrausta subsequalis Guenée, 1854 & 48 \\
\hline Pyrausta unifascialis Packard, 1873 & 57 \\
\hline
\end{tabular}


Table 1 (continued).

Taxon

\#specimens

\begin{tabular}{|l|l|}
\hline Pyrausta volupialis Grote, 1877 & 21 \\
\hline Saucrobotys fumoferalis Hulst, 1886 & 21 \\
\hline Saucrobotys futilalis Lederer, 1863 & 5 \\
\hline Scoparia biplagialis Walker, 1865 & 11 \\
\hline Sitochroa chortalis Grote, 1874 & 11 \\
\hline Sitochroa palealis (Denis \& Schiffermuller, 1775) & 3 \\
\hline Spoladea recurvalis (Fabricius, 1775) & 2 \\
\hline Tehama bonifatellus Hulst, 1887 & 236 \\
\hline Thaumatopsis fernaldella Kearfott, 1905 & 4 \\
\hline Thaumatopsis repandus Grote, 1880 & 2 \\
\hline Tosale oviplagalis Walker, 1866 & 4 \\
\hline Udea undetermined & 1 \\
\hline Udea itysalis Walker, 1859 & 7 \\
\hline Udea itysalis tillialis & 15 \\
\hline Udea profundalis Packard, 1873 & 257 \\
\hline Udea rubigalis (Guenee, 1854) & 2 \\
\hline Udea vacunalis (Grote, 1881) & 1 \\
\hline Udea washingtonalis (Grote, 1881) & 20 \\
\hline Uresiphita reversalis Guenée, 1854 & 3 \\
\hline Urola nivalis (Drury, 1773) & 1 \\
\hline & \\
\hline
\end{tabular}


Table 2

Taxon

\#specimens

Acrobasis caliginella (Hulst, 1878)

19

Acrobasis tricolorella Grote, 1878

98

Acroncosa albiflavella Barnes \& McDunnough, 1917

Aglossa cacamica Dyar, 1914

Aglossa pinguinalis (Linnaeus, 1758)

Alberada californiensis Neunzig, 1997

Ambesa laetella Grote, 1880

Ambesa walsinghami Ragonot, 1887

Amyelois transitella (Walker, 1863)

Ancylosis albipenella (Hulst, 1887)

Ancylosis morrisonella Ragonot, 1887

Ancylosis undulatella Clemens, 1860

Bandera virginella Dyar, 1908

Dasypyga alternosquamella Ragonot, 1887

Dioryctria undetermined

Dioryctria abietivorella Grote, 1878

Dioryctria albovittella Hulst, 1890

Dioryctria auranticella Grote, 1883

Dioryctria cambiicola Dyar, 1914

Dioryctria durangoensis Mutuura \& Neunzig, 1986

Dioryctria erythropasa Dyar, 1914

Dioryctria pentictonella Mutuura, Munroe \& Ross, 1969

Dioryctria ponderosae Dyar, 1914

Dioryctria pseudotsugella Munroe, 1959

1

6

25

22

5

1

55

15

1

29

54

116

21

36

48

1

1

Dioryctria rossi Munroe, 1959

Dioryctria tumicolella Mutuura, Munroe \& Ross, 1969

Ephestia elutella (Hubner, 1796)

Ephestia kuehniella Zeller, 1879

Ephestiodes gilvescentella Ragonot, 1887

Ephestiodes undetermined

Etiella zinckenella (Treitschke, 1832)

Euzophera semifuneralis Walker, 1863

74

3

219

4

4

5

24

8

Homoeosoma electella Hulst, 1887

Honora dotella Dyar, 1910

Honora mellinella Grote, 1878

Hypsopygia costalis (Fabricius, 1775)

Hypsopygia undetermined 
Table 2 (continued).

Taxon

\begin{tabular}{|c|c|}
\hline Hypsopygia phoezalis (Dyar, 1908) & 19 \\
\hline Interjectio undetermined & 17 \\
\hline Interjectio columbiella McDunnough, 1935 & 2 \\
\hline Interjectio denticulella Ragonot, 1887 & 5 \\
\hline Interjectio niviella Hulst, 1888 & 2 \\
\hline Laetilia dilatifasciella (Ragonot, 1887) & 1 \\
\hline Laetilia zamacrella Dyar, 1925 & 23 \\
\hline Lipographis fenestrella Packard, 1874 & 14 \\
\hline Macalla zelleri Grote, 1876 & 3 \\
\hline Melitara subumbiella Dyar & 11 \\
\hline Myelopsis alatella Hulst, 1887 & 1 \\
\hline Myelopsis minutularia Hulst, 1887 & 1 \\
\hline Olybria aliculella Hulst, 1887 & 3 \\
\hline Oneida undetermined & 3 \\
\hline Phobus brucei Hulst, 1895 & 5 \\
\hline Phobus curvatella Ragonot, 1887 & 6 \\
\hline Phycitodes albatella (Ragonot, 1887) & 2 \\
\hline Phycitodes mucidella (Ragonot, 1887) & 4 \\
\hline Phycitodes reliquellum (Dyar, 1904) & 17 \\
\hline Pima albiplagiatella Packard, 1874 & 2 \\
\hline Pima fergusoni Neunzig, 2003 & 3 \\
\hline Pima fosterella Hulst, 1888 & 10 \\
\hline Pima occidentalis Heinrich, 1956 & 2 \\
\hline Plodia undetermined & 22 \\
\hline Plodia interpunctella (Hubner, 1813) & 45 \\
\hline Pococera aplastella Hulst, 1888 & 1 \\
\hline Promylea lunigerella Ragonot, 1887 & 2 \\
\hline Pyla aeneoviridella Ragonot, 1887 & 28 \\
\hline Pyla aenigmatica Heinrich, 1956 & 19 \\
\hline Pyla fusca Haworth, 1811 & 45 \\
\hline Pyla undetermined & 24 \\
\hline Pyla scintillans Grote, 1881 & 4 \\
\hline Pyla westerlandi Wilterding \& Balogh, 2002 & 5 \\
\hline Pyralis farinalis (Linnaeus, 1758) & 58 \\
\hline Rostrolaetilia minimella Blanchard \& Ferguson, 1975 & 2 \\
\hline Sarata caudellella Dyar, 1904 & 1 \\
\hline
\end{tabular}


Table 2 (continued)

Taxon \#specimens
\begin{tabular}{|l|l|}
\hline Sarata edwardsialis Hulst, 1886 & 1 \\
\hline Sarata incanella Hulst, 1895 & 2 \\
\hline Sarata perfuscalis Hulst, 1886 & 4 \\
\hline Sarata pullatella Ragonot, 1887 & 12 \\
\hline Sciota undetermined & 15 \\
\hline Sciota levigatella (Hulst, 1892) & 1 \\
\hline Sciota tarmitalis (Hulst, 1886) & 4 \\
\hline Toripalpus breviornatalis Grote, 1878 & 2 \\
\hline Toripalpus trabalis Grote & 23 \\
\hline Vitula edmandsii (Packard, 1865) & 25 \\
\hline Vitula pinei Heinrich, 1956 & 6 \\
\hline Vitula undetermined & 29 \\
\hline Zophodia grossulariella (Hubner, 1809) & 5 \\
\hline Pyralidae: Phycitinae undetermined & 24 \\
\hline
\end{tabular}

Additional material from outside of North America is present, but was not digitized and catalogued at this time.

Label data for these specimens can be accessed via online data portals that serve osac IPT data (e.g., www.gbif.org) or by direct download via the archived datasets stored in the supplemental files of this paper.

\section{Discussion:}

Minor corrections and other small modifications made in the future to this dataset will be accomplished by issuing a new version and providing a file of modified records to the supplemental files. Major additions, such as new records, new data fields, new contributors or other significant modifications will be accomplished through the generation and release of new datasets.

\section{References}

Moth Photographers Group (2019) Digital Guide to Moth Identification. https: / / mothphotographersgroup.msstate.edu/ [accessed April 1, 2019.2019]

Seltmann, K. C. et al. 2017. LepNet: The Lepidoptera of North America Network. Zootaxa 4247 (1): 073-077. https: / / doi.org/10.11646/ zootaxa.4247.1.10.

Shepard J.H., C.J. Marshall, P.C. Hammond. 2018. North American Lepidoptera in the Oregon State Arthropod Collection: Hesperiidae, Pieridae, Papilionidae. Catalog: Oregon State Arthropod Collection 2(2): 1-2 and supplement file. doi: $\underline{\text { http: / / dx.doi.org/10.5399/osu/cat osac.2.2.4480 }}$ 